\title{
Optical Inclinometer Based on a Phase-Shifted Bragg Grating in a Taper Configuration
}

\author{
João P. Moura, Susana O. Silva, Martin Becker, M. Rothhardt, Hartmut Bartelt, \\ José Luís Santos, and Orlando Frazão
}

\begin{abstract}
A simple optical inclinometer based on a phaseshifted Bragg grating in a taper configuration is proposed. Two phase-shifted fiber Bragg gratings were fabricated using a DUV femtosecond laser technique in the taper region, with taper waist diameters of $30 \mu \mathrm{m}$ and $50 \mu \mathrm{m}$. Both sensing heads were compared based on their response to angle and strain. Whereas the higher diameter sensor yielded a higher sensitivity to curvature $(23.8 \pm 0.3 \mathrm{pm} /$ degree $)$, the lower diameter one was more sensitive to strain $(8.94 \pm 0.04 \mathrm{pm} / \mu \varepsilon)$.
\end{abstract}

Index Terms-Optical fiber sensors, Bragg gratings, Taper.

\section{INTRODUCTION}

$\mathbf{M}$ OST FIBER Bragg gratings (FBG) designed for practical applications are non-uniform grating structures. Often the main reason for choosing a non-uniform design is to reduce the undesirable side lobes prevalent in uniform-grating spectra. However, many other optical properties of fiber gratings can be adjusted by tailoring the grating parameters along the fiber axis. Sharp, well-defined filter shapes are rapidly becoming critical characteristics for passive components in dense wavelength division multiplexed (DWDM) communication systems. On the other hand, chirping the period of a grating enables the dispersive properties of scattered light to be tailored [1].

Sometimes it is desirable to create discrete, localized phase shifts in an otherwise periodic grating. Discrete phase shifts can be used to open an extremely narrow transmission resonance in a reflection grating or to tailor the passive filter shape. The principle of the phase shift was demonstrated by Alfemess et al. [2] in periodic structures made from semiconductor materials, where a phase shift was introduced by etching a space at the center of the device. This forms the basis of the single-mode phase-shifted semiconductor DFB laser [3]. A similar device may be constructed in optical fibers using

Manuscript received May 28, 2013; revised November 21, 2013; accepted December 9, 2013. Date of publication December 18, 2013; date of current version January 24, 2014. This work was supported in part by the ERDF - European Regional Development Fund through the COMPETE Programme and in part by FCT - Fundação para a Ciência e a Tecnologia Portuguese Foundation for Science and Technology under Project FCOM-01-0124-FEDER022701.

J. P. Moura and J. L. Santos are with INESC Porto, Porto 4200-465, Portugal, and also with the Department Física e Astronomia, Faculdade de Ciências, Universidade do Porto, Porto 4099-002, Portugal (e-mail: jpmoura@fc.up.pt; josantos@fc.up.pt).

S. O. Silva and O. Frazão are with INESC Porto, Porto 4200-465, Portugal (e-mail: sfsilva@inescporto.pt; ofrazao@inescporto.pt).

M. Becker, M. Rothhardt, and H. Bartelt are with the Institute of Photonic Technology, Jena 07745, Germany (e-mail: martin.becker@ipht-jena.de; hartmut.bartelt@ipht-jena.de).

Color versions of one or more of the figures in this letter are available online at http://ieeexplore.iee.org.

Digital Object Identifier 10.1109/LPT.2013.2295323 various techniques. Such processing produces two gratings out of phase with each other, which act as a wavelength-selective Fabry-Pérot resonator. The resonant wavelength corresponds to a transmission filter over the rejection band of the Bragg grating and depends on the amplitude and location of the phase change. The resonant wavelength of this filter can be tuned over the spectral response of the Bragg grating by introducing one or several phase shifts within the range $[0-2 \pi]$ in particular locations along the grating length [4]. This technique allows the exploitation of Bragg gratings' spectral response in transmission which can be used for specific applications, namely, in communication and sensing.

In terms of optical sensing, phase-shifted FBGs have been used to measure parameters such as vibration [5], strain [6] and pressure [7], [8] with increased sensitivity in relation to conventional FBG systems.

By tapering a standard single-mode fiber, the evanescent field of light propagating in the core can be coupled to the external environment [9], enabling applications such as chemical or biological sensing [10]. In particular, tapers can be used to measure angles due to an intensity loss of the evanescent field when the taper is bent [11]. Taper structures can be achieved by etching the fiber cladding or by lengthening a portion of fused fiber. Gratings can then be fabricated in the tapered region resulting in enhanced properties, for example for strain sensing [12].

In this letter, the authors present an optical inclinometer based on phase-shifted Bragg gratings in a taper topology. The phase-shifted FBG is fabricated through a DUV femtosecond laser technique in the taper region. The sensing head is characterized for different angles and also to strain.

\section{EXPERIMENTAL SETUP}

To develop the phase-shifted FBG-based sensing heads, a $2 \mathrm{~mm}$ wide slit was placed on a translation stage with an adjustable mirror in a conventional FBG fabrication setup based on the DUV femtosecond laser technique to perform a $2.5 \mathrm{~mm}$ displacement between FBG inscriptions. Each phaseshifted FBG was based on two FBGs written in the waist section of a tapered single-mode fiber. The taper region was fabricated using a VYTRAN glass processing platform which heats a portion of the fiber and pulls the ends of the heated segment [10]. Using the described method two tapered phaseshifted FBGs were fabricated, both with a total taper length of approximately $45 \mathrm{~mm}$, differing only in taper waist diameter: one with $30 \mu \mathrm{m}$ and the other with $50 \mu \mathrm{m}$.

To perform the sensor interrogation, a $100 \mathrm{~nm}$ broadband source centered at $1550 \mathrm{~nm}$ and an OSA with a maximum 


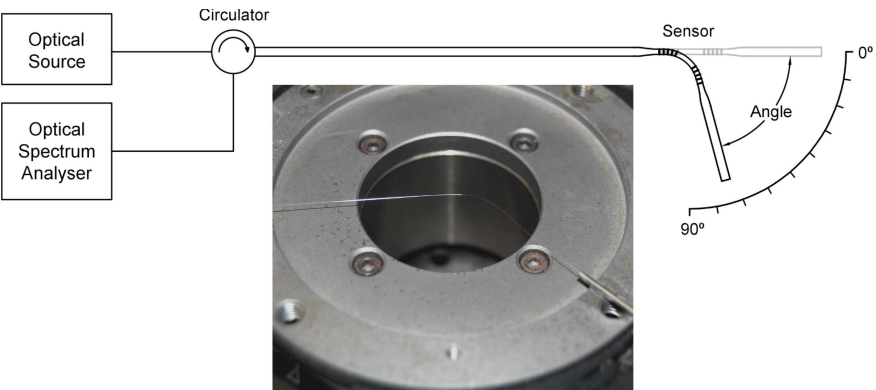

Fig. 1. Scheme of the angular curvature characterization setup and detailed photo of a bent inclinometer.
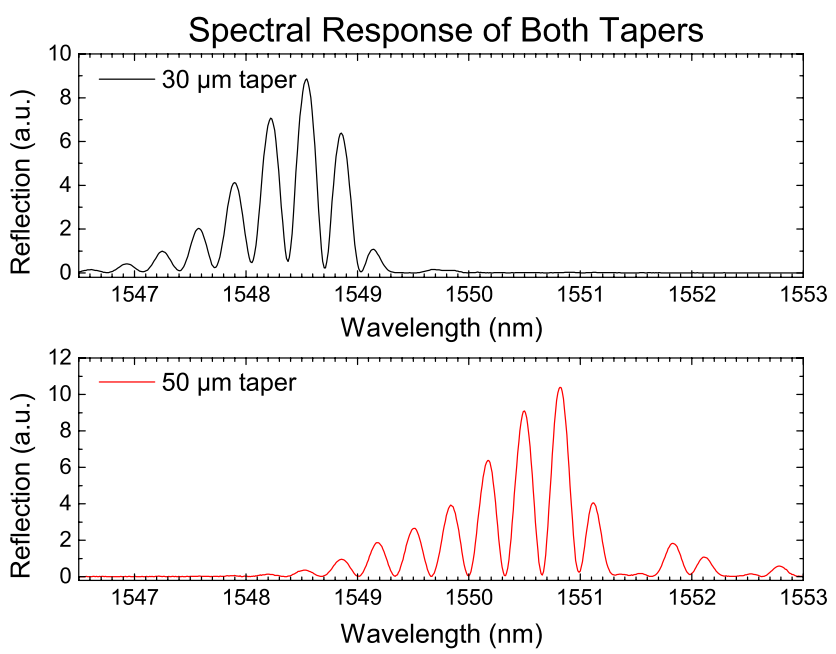

Fig. 2. Optical spectra of two phase-shifted FBGs inscribed on tapered single mode fibers with $30 \mu \mathrm{m}$ and $50 \mu \mathrm{m}$ taper waist diameters.

resolution of $0.01 \mathrm{~nm}$ were connected to the sensor through an optical circulator device to analyze the reflection response of the optical inclinometer. To measure the relationship between the spectrum and curvature, the sensors were set on a rotating platform with an uncertainty of 0.5 degrees. One end was stuck to a fixed position whereas the other was rotated between 0 and 90 degrees. Considering only the taper region is bent and supposing a circular arc geometry for the bending, the curvature radius is given by the taper length divided by the angle, which in our case corresponds to a range between 0 and $2.9 \mathrm{~cm}$ and a curvature between 0 and $35 \mathrm{~m}^{-1}$. The setup scheme for angular characterization can be seen in Fig. 1, as well as a picture of a bent sensor. For strain characterization, the sensing heads were placed on micrometric translation stages to control the subjected stretching.

\section{Results AND Discussion}

Both sensing heads present high reflectivities showing several peaks, as expected [13], each $0.3 \mathrm{~nm}$ apart and with a FWHM of $0.2 \mathrm{~nm}$. Fig. 2 depicts the spectral response of both sensing heads with an envelope FWHM of $1 \mathrm{~nm}$.

For angular characterization, the sensing heads were interrogated in steps of 5 degrees between 0 and 90 degrees. When the sensing head is subjected to curvature the taper region is deformed, an effect that alters the phase-shifted FBG properties: the spectrum is redshifted and its intensity decreases [see Fig. 3(a)]. The intensity loss is related with

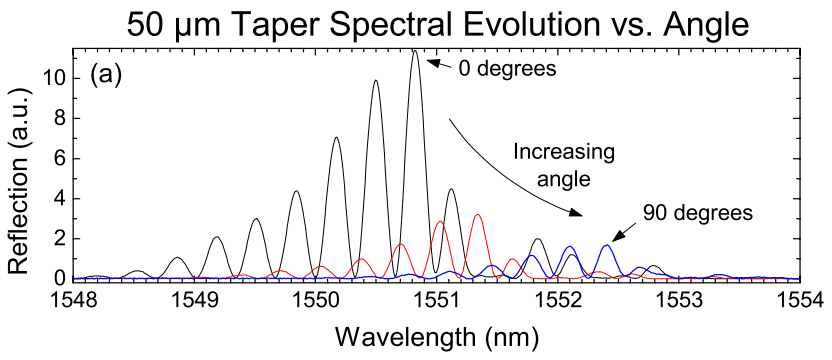

$30 \mu \mathrm{m}$ Taper Spectral Evolution vs. Strain

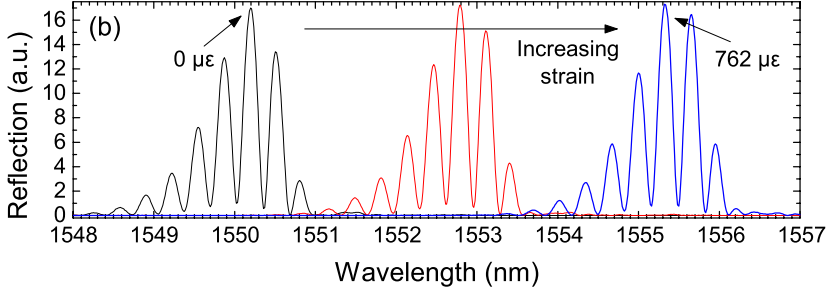

Fig. 3. Spectral response of both tapered phase-shifted FBGs in response to angle (a) and strain (b).

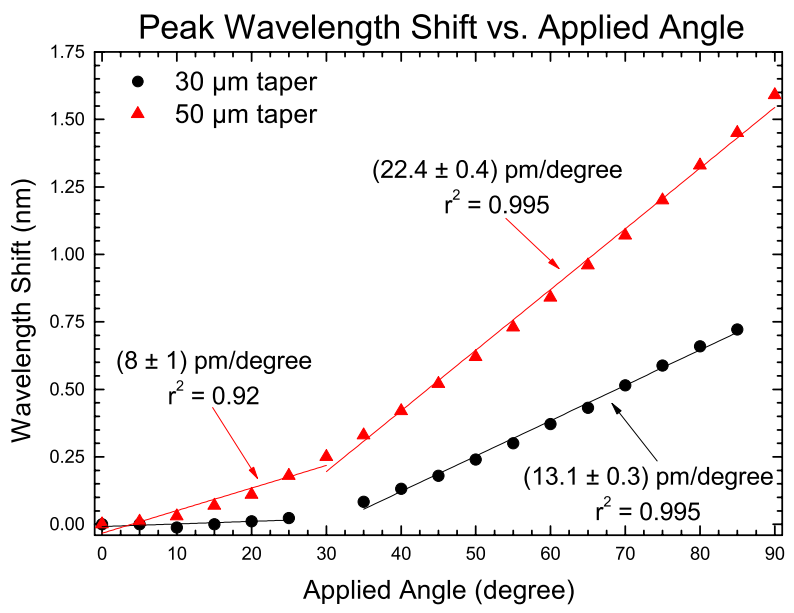

Fig. 4. Peak wavelength shift as a function of the applied angle for both sensors. Slopes and $\mathrm{r}^{2}$ parameters are indicated for different linear regimes.

the fact that the taper is non adiabatic which results in a high coupling of light that propagates within the fiber core with the cladding. If the taper is bent, the light in the cladding region is lost to the external environment. The spectral redshift has a different origin. When a standard fiber with a phase-shifted FBG is bent, the neutral axis coincides with the geometrical axis of the fiber. Thus, no strong spectral dependency on curvature is expected. In the tapered case, if the curvature forces result in a non-ideal bending, for example due to irregularities in the taper structure, the geometrical core may be radially pushed away from the neutral axis. The gratings then suffer net positive tension, resulting in the observed spectral redshift.

Fig. 4 shows the wavelength response to tilting for both sensors where two linear regimes can be identified. For angles lower than 30 degrees, the $50 \mu \mathrm{m}$ sensor has a sensitivity of $8 \pm 1 \mathrm{pm} /$ degree $\left(\mathrm{r}^{2}=0.92\right)$. For angles higher than 30 degrees, the $50 \mu \mathrm{m}$ taper has a sensitivity of $22.4 \pm 0.4 \mathrm{pm} /$ degree $\left(\mathrm{r}^{2}=0.995\right)$ and the one with $30 \mu \mathrm{m}$ has a sensitivity of $13.1 \pm 0.3 \mathrm{pm} /$ degree $\left(\mathrm{r}^{2}=0.995\right)$. It should be noted that the 


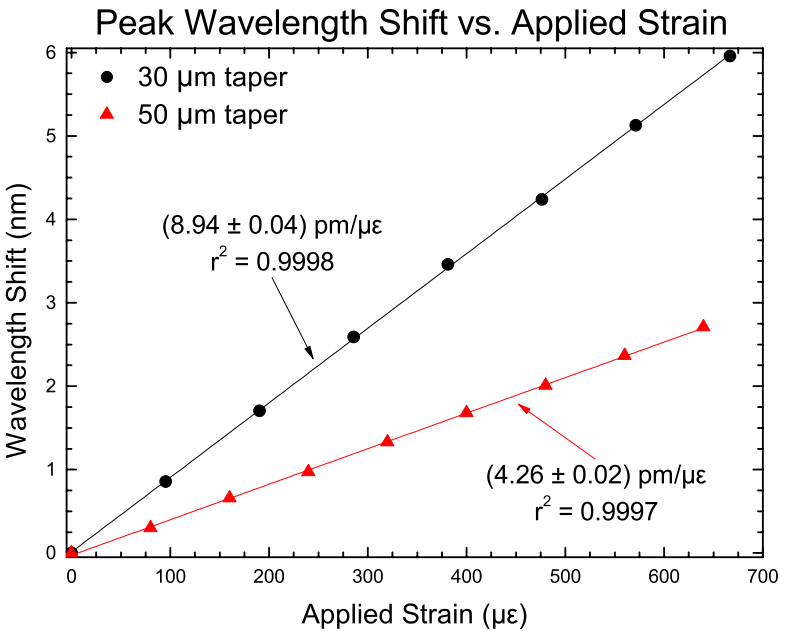

Fig. 5. Peak wavelength shift as a function of applied strain for both sensors. Slopes and $\mathrm{r}^{2}$ parameters are indicated for different linear regimes.

thicker $50 \mu \mathrm{m}$ sensor has a higher sensitivity than the thinner $30 \mu \mathrm{m}$ one. For a complete study of the sensitivity dependency on the diameter one should analyze this effect on a wider range of diameters. With such a small set of samples it is not possible to define a clear model to this problem. In future research, special attention should be paid to the taper structure, for differences in geometry could also be the source of stronger non-ideal bending effects.

When strain is applied without curvature, previous works have shown that FBGs in tapers have a much stronger response in wavelength than FBGs in standard SMFs. This result is expected taking into account that strain $\epsilon$ is related with tension $T$ (force divided by area) by $\epsilon=T / E$, where $E$ is Young's modulus. Since the cross section area of the taper region is lower than that of the SMF28, for the same applied force the tension is higher for the taper. Consequently, tapers suffer a higher strain which effectively results in a higher strain sensitivity of the whole sensing system [12]. Fig. 3(b) shows the spectral evolution and Fig. 5 shows the peak wavelength shift when strain is applied to the sensing heads. The measured strain sensitivities were $8.94 \pm 0.04 \mathrm{pm} / \mu \varepsilon\left(\mathrm{r}^{2}=0.9998\right)$ and $4.26 \pm 0.02 \mathrm{pm} / \mu \varepsilon\left(\mathrm{r}^{2}=0.9997\right)$ for the $30 \mu \mathrm{m}$ and $50 \mu \mathrm{m}$ diameter tapers, respectively. Both sensitivities are high when compared with conventional FBG sensors [14].

The sensor spectral dependence on temperature is expected to be similar to that of an FBG in a standard SMF as published in the literature [14]. Previous works have shown that an FBG response to temperature is solely related with the thermal expansion of the cristal that constitutes the fiber and the refractive index variation with temperature. Therefore it is geometry independent with sensitivities of the order of $10 \mathrm{pm} /{ }^{\circ} \mathrm{C}$ [9].

\section{CONCLUSION}

Phase-shifted FBGs where fabricated in tapered topologies in standard single-mode fibers. This type of structure effectively increases the sensor sensitivity when it is subjected to strain. The tapers where fabricated by elongating a heated fiber segment while the gratings where developed using a DUV femtosecond laser technique. Two sensing heads with different taper diameters where characterized to strain and angle.

When bent, the wider taper $(50 \mu \mathrm{m})$ showed a higher sensitivity of $22.4 \pm 0.4 \mathrm{pm} /$ degree in comparison to the thinner one $(30 \mu \mathrm{m})$ with $13.1 \pm 0.3 \mathrm{pm} /$ degree in a range between 30 and 90 degrees. Future studies are being idealized to physically interpret this effect.

For no bending, the strain sensitivity of the $50 \mu \mathrm{m}$ taper $(4.26 \pm 0.02 \mathrm{pm} / \mu \varepsilon)$ was lower than the $30 \mu \mathrm{m}$ taper $8.94 \pm$ $0.04 \mathrm{pm} / \mu \varepsilon$. Thinner tapers have a smaller cross section and therefore give rise to sensors with higher sensitivity to strain.

Finally, a new sensing head can be proposed based on two different tapers with phase-shifted FBGs in series and it should be possible to use it for simultaneous measurement of strain and curvature. Be it in series or by itself, this type of structure can be used in several applications, namely in civil or aeronautical engineering for curvature or inclination sensing, in which case the sensor interrogation can be performed with cheaper and more practical techniques than an OSA, such as tunable optical filtering or interferometry [15].

\section{REFERENCES}

[1] H. Kogelnik, "Filter response of nonuniform almost-periodic structures," Bell Syst. Tech. J., vol. 55, no. 1, pp. 109-126, Jan. 1976.

[2] R. C. Alferness, C. H. Joyner, M. D. Divino, M. J. R. Martyak, and L. L. Buhl, "Narrowband grating resonator filters in InGaAsP/InP waveguides," Appl. Phys. Lett., vol. 49, no. 3, pp. 125-127, Jul. 1986.

[3] K. Utaka, S. Akiba, K. Skai, and Y. Matsushima, " $\lambda / 4$-shifted InGaAsP/InP DFB lasers," IEEE J. Quantum Electron., vol. 22, no. 7, pp. 1042-1051, Jul. 1986.

[4] G. P. Agrawal and S. Radic, "Phase-shifted fiber Bragg gratings and their application for wavelength demultiplexing," IEEE Photon. Technol. Lett., vol. 6, no. 8, pp. 995-997, Aug. 1994.

[5] A. I. Azmi, D. Sen, W. Sheng, J. Canning, and G.-D. Peng, "Performance enhancement of vibration sensing employing multiple phase-shifted fiber Bragg grating," J. Lightw. Technol., vol. 29, no. 22, pp. 3453-3460, Nov. 15, 2011.

[6] D. Gatti, G. Galzerano, D. Janner, S. Longhi, and P. Laporta, "Fiber strain sensor based on a $\Pi$-phase-shifted Bragg grating and PoundDrever-Hall technique," Opt. Express, vol. 16, no. 3, pp. 1945-1950, 2008.

[7] T. Liu and M. Han, "Analysis of П-phase-shifted fiber Bragg gratings for ultrasonic detection," IEEE Sensors J., vol. 12, no. 7, pp. 2368-2373, Jul. 2012.

[8] S. E. U. Lima, et al., "Fiber laser sensor based on a phase-shifted chirped grating for acoustic sensing of partial discharges," Photon. Sensors, vol. 3 , no. 1 , pp. $44-51,2013$.

[9] S. F. O. Silva , L. A. Ferreira , F. M. Araújo, J. L. Santos, and O. Frazäo, "Fiber Bragg grating structures with fused tapers," Fiber Integr. Opt., vol. 30, no. 1, pp. 9-28, 2011.

[10] S. Silva, et al., " $\mathrm{H}_{2}$ sensing based on a Pd-coated tapered-FBG fabricated by DUV femtosecond laser technique," IEEE Photon. Technol. Lett., vol. 25, no. 4, pp. 401-403, Feb. 15, 2013.

[11] L. C. Bobb, P. M. Shankar, and H. D. Krumboltz, "Bending effects in biconically tapered single-mode fibers," J. Lightw. Technol., vol. 8, no. 7 , pp. 1084-1090, Jul. 1990.

[12] O. Frazão, S. Silva, A. Guerreiro, J. Santos, L. Ferreira, and F. Araújo, "Strain sensitivity control of fiber Bragg grating structures with fused tapers," Appl. Opt., vol. 46, no. 36, pp. 8578-8582, Dec. 2007.

[13] M. Becker, et al., "Fiber Bragg grating inscription combining DUV subpicosecond laser pulses and two-beam interferometry," Opt. Express, vol. 16, no. 23, pp. 19169-19178, Nov. 2008.

[14] A. D. Kersey, et al., "Fiber grating sensors," J. Lightw. Technol., vol. 15, no. 8, pp. 1442-1463, Aug. 1997.

[15] J. Chen, B. Liu, and H. Zhang, "Review of fiber Bragg grating sensor technology," Frontiers Optoelectron. China, vol. 4, no. 2, pp. 204-212, Jun. 2011. 